\title{
Acknowledgment to Reviewers of Hydrology in 2020
}

\author{
Hydrology Editorial Office
}

Citation: Hydrology Editorial Office. Acknowledgment to Reviewers of Hydrology in 2020. Hydrology 2021, 8 , 18. https://doi.org/10.3390/ hydrology 8010018

Published: 27 January 2021

Publisher's Note: MDPI stays neutral with regard to jurisdictional claims in published maps and institutional affiliations.

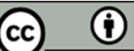

Copyright: (c) 2021 by the author. Licensee MDPI, Basel, Switzerland. This article is an open access article distributed under the terms and conditions of the Creative Commons Attribution (CC BY) license (http://creativecommons.org/licenses /by/4.0/).

MDPI AG, St. Alban-Anlage 66, 4052 Basel, Switzerland

Peer review is the driving force of journal development, and reviewers are gatekeepers who ensure that Hydrology maintains its standards for the high quality of its published papers. Thanks to the cooperation of our reviewers, in 2020, the median time to first decision was 13 days and the median time to publication was 38 days. The editors would like to express their sincere gratitude to the following reviewers for their precious time and dedication, regardless of whether the papers were finally published:

Abeynayaka, Amila

Addamo, Anna M.

Afshar, Mehdi $\mathrm{H}$.

Aga, Alemu O.

Ahmad, Qurat Ul Ain

Ahmadi, Behzad

Ahmed, Farzana

Akbariyeh, Simin

Akmalov, Shamshodbek

Alexakis, Dimitrios E.

Al-Faraj, Furat

Allen, Melissa Ree

Al-Madhhachi, Abdul-Sahib

Al-mamari, Mahmood M.

Al-Murib, Muhanned D.

Amanambu, Amobichukwu

Anderson, Ray

Apollaro, Carmine

Apollonio, Ciro

Argaman, Eli

Arnone, Elisa

Aryal, Yog

Atique, Usman

Auler, Augusto

Avila, Alvaro Javier

Aygün, Okan

Ayzel, Georgy

Baba, Wassim Mohamed

Babovic, Vladan

Bailey, Ryan T.

Bajić, Dragoljub

Ball, Tom

Baltas, Evangelos

Barańczuk, Jacek

Barber, Michael Ernest

Barberes, Gabriel
Barbieri, Maurizio

Barešić, Jadranka

Barsanti, Michele

Bazo, Juan

Beceiro, Paula

Beranová, Romana

Birylo, Monika

Blade, Ernest

Blanco-Ward, Daniel

Błaś, Marek

Boavida, Isabel

Bokuniewicz, Henry J.

Bonacci, Ognjen

Boudhar, Abdelghani

Boyce, Scott E.

Bray, Erin

Brilly, Mitja

Bujakowski, Filip

Burlakovs, Juris

Burt, Peter

Bus, Agnieszka

Bustos-Terrones, Yaneth

Cai, Jialiang

Canales, Fausto

Capraro, Flavio

Cash, Benjamin A.

Cerasoli, Sofia

Cervi, Federico

Chang, Ching-Fu

Chanzy, Andre

Chau, Kwok-Wing

Chen, Wei-Bo

Chen, $\mathrm{Xi}$

Cheng, Weiming

Chishtie, Farrukh

Cho, Huidae 
Cho, Younghyun

Chou, Eric Ting-Kuei

Chouaib, Wafa

Clara Fava, Maria

Colaiuda, Valentina

Colombo, Loris

Colombo, Nicola

Confesor, Remegio

Conticello, Federico Rosario

Costanzo, Carmelina

Cüneyd Demirel, Mehmet

Czerwionka, Krzysztof

Da Fonseca, André Ribeiro

Dąbrowska, Dominika

Daneshvar, Fariborz

Dang, Thanh Duc

Dannowski, Ralf

Darabi, Hamid

Dawes, Warrick R.

De La Casa, Antonio

De La Fuente, Alberto

De Lima, Isabel Pedroso

De Lurdes Dinis, Maria

De Morais, Marcos Vinicius Bueno

De Vita, Pantaleone

Deb, Proloy

Delle Rose, Marco

Demertzi, Kleoniki

Demlie, Molla B.

Detert, Martin

Dhakal, Nirajan

Di Curzio, Diego

Di Giuseppe, Dario

Di Matteo, Lucio

Díaz Delgado, Carlos

Dietrich, Ottfried

Dimitriadis, Panayiotis

Dimuccio, Luca

Ding, Yongjian

Do Amaral Cunha, Ana Paula Martins

Dobre, Mariana

Domingo-Pinillos, Juan Carlos

Dorea, Caetano

Dornelles, Fernando

Doro, Kennedy

Dougherty, Erin

Doulgeris, Charalampos

Dragovich, Deirdre

Duan, Weili

Dubinina, Elena

Duffy, Walter G.
Duncan, Emily

Durán-Valsero, Juan José

Đurin, Bojan

Durowoju, Olatunde S.

Dvory, Noam Zach

Ehsan Bhuiyan, Md Abul

Eliades, Marinos

Elosegi, Arturo

ElSaadani, Mohamed

Eltarabily, Mohamed

Escobar, Yesid Carvajal

Espinoza, Carlos Zúñiga

Essenfelder, Arthur

Ezzeddine, Zeinab

Faccini, Francesco

Fan, Jinlong

Farmer, William $\mathrm{H}$.

Fernández-Aracil, Patricia

Fischer, Christine

Fitzgibbon, John

Fonley, Morgan

Frappart, Frédéric

Gadedjisso-Tossou, Agossou

Galagedara, Lakshman

Gampe, David

Gangrade, Sudershan

Ganske, Anette

Garee, Khan

Garrote Revilla, Julio

Gautam, Mahesh

Ge, Yingchun

Gell, Peter A

Gentilucci, Matteo

Georgiou, Pantazis E.

Ghafouri-Azar, Mona

Giacometti, Andrea

Gierlak, Piotr

Gilewski, Paweł

Gilewski, Paweł Grzegorz

Gioia, Andrea

Gitz, Dennis C.

Giudicianni, Carlo

Golian, Saeed

Goodrich, Gregory B.

Gorgoglione, Angela

Gourbesville, Philippe

Granata, Francesco

Greco, Michele

Grinevsky, Sergey O

Grochowska, Jolanta

Gurwin, Jacek 
Gutiérrez López, Alfonso

Gutierrez Lopez, Jose

Hamedi, Amirmasoud

Hanich, Lahoucine

Harte, Philip T.

Hatchett, Benjamin J.

Haver, Darren L.

Hawley, Robert

$\mathrm{He}$, Siwei

$\mathrm{He}$, Yong

Heddam, Salim

Hejduk, Leszek

Heo, Jun-Haeng

Herrera, Miguel Angel

Hewson, Michael

Hofman, Jan

Hong, Nian

Horvat, Bojana

Hosseiny, Hossein

Høyer Svendsen, Synne

Hsieh, Ping-Cheng

Hssaisoune, Mohammed

$\mathrm{Hu}$, Caihong

Huang, Tianming

Hussain, Yawar

Hutorowicz, Andrzej

Iasiello, Marcello

Ioana-Toroimac, Gabriela

Iosub, Marina

Jabbari, Aida

Javeed, Nadeem

Jawecki, Bartosz

Jiang, Jianhua

Jiménez-Madrid, Alberto

Jones, Ian C.

Jones, Norm

Jost, Anne

Juana Sirgado, Luis

Jukić, Damir

Júnez Ferreira, Hugo Enrique

Kadir, Tariq

Kagone, Stefanie

Kałuża, Tomasz

Kambezidis, Harry

Kamis, Ahmed Samy

Kanakoudis, Vasilis

Kanduč, Tjaša

Karabil, Sitar

Karakouzian, Moses

Karleuša, Barbara

Kasperska-Wołowicz, Wiesława
Katzourakis, Vasileios

Kazama, Shinobu

Kharismalatri, Hefryan Sukma

Kim, Changhwan

Kim, Dongkyun

Kinzel, Carolina

Klamerus-Iwan, Anna

Kochanek, Krzysztof

Kociuba, Waldemar

Kolerski, Tomasz

Kollet, Stefan

Kong, Yanlong

Konstantakopoulou, Foteini

Križan, Peter

Kumar, Pankaj

Kuriqi, Alban

Kuzevič, Štefan

Labat, David

Labate, Antonino

Lade, Harshad S.

Lang, Štěpán

Lange, Manfred

Lazzari, Maurizio

Li, Hui

Li, Qiang

Li, Rui

Li, Xiaodong

Liotta, Marcello

Lipiński, Seweryn

Litt, Guy

Liu, Changjun

Liu, Jih-Shun

Liu, Ronghua

Liu, Tao

Liu, Tie

Lo, Kwong Fai Andrew

Loukas, Athanasios

Luque-Espinar, Juan A.

Ma, Yaoming

Mahmood, Taufique

Mahmud, M. Rizaludin

Malekian, Arash

Manfreda, Salvatore

Marinello, Francesco

Marteau, Baptiste

Martinat, Stanislav

Mastronuzzi, Giuseppe

Masumoto, Yukio

Mateo-Lázaro, Jesús

Matos Silva, Maria

Mazza, Alessandro 
McMartin, Dena

Medici, Giacomo

Mekhonoshin, Aleksey

Memon, Muhammad Sohail

Mendoza, Rose Marie O.

Michelon, William

Miglio, Antonio

Millares, Agustín

Mirás-Avalos, José Manuel

Młynski, Dariusz

Mohammadi, Babak

Molot, Lewis

Morosanu, Gabriela

Muhuri, Arnab

Musayev, Sardorbek

Nabil, Bachagha

Najibi, Nasser

Nania, Leonardo

Napolitano, Francesco

Nardi, Fernando

Nawrot, Adam P.

Nedjai, Rachid

Nertan, Argentina

Niaghi, Ali Rashid

Nicolis, Orietta

Nicu, Ionut Cristi

Nippgen, Fabian

Nofal, Salah

Oh, Sungmin

Oikonomou, Panagiotis D.

Omani, Nina

Onaca, Alexandru

Onyutha, Charles

Operacz, Agnieszka

Ostovari, Yaser

Paerl, Hans W.

Pajak, Katarzyna

Palermo, Stefania Anna

Pan, Jinmei

Pan, Tsung-Yi

Panagoulia, Dionysia

Papalexiou, Simon Michael

Park, Daeryong

Park, Mincheol

Parlov, Jelena

Partsinevelos, Panagiotis

Pasculli, Antonio

Pavlic, Kresimir

Pavlovskii, Igor

Paz, Igor

Pech, Pavel
Peña, Carlos

Peña-Haro, Salvador

Pérez Pastor, Alejandro

Petaccia, Gabriella

Peterson, Eric W.

Pfannerstill, Matthias

Piacentini, Daniela

Piccini, Leonardo

Pisaturo, Giuseppe Roberto

Pliakas, Fotios-Konstantinos

Pochwat, Kamil

Polykretis, Christos

Pradhanang, Soni M.

Principato, Francesca

Ptak, Mariusz

Pueppke, Steven

Qin, Dajun

Quej, Victor H.

Quintanar, Arturo

Racar, Marko

Rajulapati, Chandra Rupa

Ramadas, Meenu

Ramage, Joan

Reza, Arif

Rianna, Guido

Ricci, Giovanni Francesco

Rienzi, Eduardo A.

Risch, Eric

Rodrigo-Clavero, María Elena

Rodrigo-Comino, Jesus

Romano, Emanuele

Rosso, Maurizio

Rozos, Evangelos

Rubinić, Josip

Ruescas, Ana B.

Rymuza, Katarzyna

Saavedra, Oliver C.

Saha, Gopal Chandra

Saha, Korak

Salazar, Juan F.

Salvati, Luca

Sample, David

Sampson, David

Santonastaso, G. F.

Santos, Ricardo

Sapač, Klaudija

Sarma, S.S.S.

Schiattarella, Marcello

Schmidt, Sabine

Scholz, Miklas

Segoni, Samuele 
Seidel, Chad

Sellami, Haykel

Şerban, Gheorghe

Shabani, Afshin

Shangguan, Ziheng

Sheih, Yuch

Shen, Shui-Long

Shoaib, Muhammad

Sidle, Roy C.

Sidorchuk, Aleksey

Simeone, Vincenzo

Simonneaux, Vincent

Simpson, Greg D.

Skre, Oddvar

Słowik-Opoka, Ewa

Soldatenko, Sergei A.

Song, Xuehang

Soto, David X

Soulis, Konstantinos

Spiliotopoulos, Marios

Sprenger, Christoph

Springer, Gregory S.

Stancalie, Gheorghe

Steenhuis, Tammo

Stepanova, N Yu

Stetler, Larry D.

Storto, Andrea

Strati, Virginia

Struminska-Parulska, Dagmara I.

Sudol, Miroslaw

Sun, Shanlei

Sun, Shuangke

Szabó-Takács, Beáta

Szafarczyk, Anna

Szatyłowicz, Jan

Szewczak, Kamil

Talchabhadel, Rocky

Tamaddun, Kazi

Tanaka, Tomohiro

Tananaev, Nikita

Tebakari, Taichi

Tegegne, Getachew

Telesca, Vito

Thornton, James

Thornton, Jeffrey A.

Tilak, Amey S.

Tomoyoshi, Nishimura

Toro, Norman

Totaro, Vincenzo

Tucciarelli, Tullio

Turconi, Laura
Ulloa, Hugo

Vaittinada Ayar, Pradeebane

Valipour, Mohammad

Vecchio, Antonio

Venetsanou, Panagiota

Venkataraman, Kartik

Veról, Aline

Vespasiano, Giovanni

Voda, Mihai

Volpi, Elena

Voutos, Yorghos

Vyshkvarkova, Elena V.

Wagner, Beatrice

Wallace, Corey

Wannous, Manal

Wartalska, Katarzyna

Wasilkowski, Daniel

Webster, Clare

Wetzel, Karl-Friedrich

Williams, Gustavious Paul

Wirmvem, Mengnjo Jude

Wojciechowski, Tomasz

Woldemariam, Gezahegn Weldu

Wolski, Piotr

Wolter, Calvin

Wright, Steven J.

Wróżyński, Rafał

Wu, Shiangjen

$\mathrm{Xu}$, Ximeng

Xuan, Yunqing

Yang, Jeong Seok

Yang, Xiaohua

Yannopoulos, Stavros I.

Yapiyev, Vadim

Yettella, Vineel

Yi, Shuang

Yin, Xiaobin

Yin, Zhenliang

Yoon, Seong-Sim

Youssef, Ahmed M.

Yuan, Ruiqiang

Zamfir, Rares Halbac-Cotoara

Zdon, Andy

Zelenakova, Martina

Zema, Demetrio Antonio

Zeroual, Ayoub

Zhang, Hao

Zhang, Xinxuan

Zheng, Xing

Zheng, Zicheng

Zhu, Laiyin 